\title{
Effect of icariin on fracture healing in an ovariectomized rat model of osteoporosis
}

\author{
HONG CAO $^{1 *}$, YING ZHANG ${ }^{2 *}$, WEI QIAN ${ }^{1}$, XIAO-PENG GUO ${ }^{3}$, CHEN SUN $^{1}$, \\ LEI ZHANG ${ }^{1}$ and XIN-HUA CHENG ${ }^{1}$ \\ ${ }^{1}$ Department of Orthopedic Surgery; ${ }^{2}$ Reproductive Medicine Center, Renmin Hospital, Hubei University of Medicine; \\ ${ }^{3}$ Department of Orthopedic Surgery, Taihe Hospital, Hubei University of Medicine, Shiyan, Hubei 442000, P.R. China
}

Received June 21, 2015; Accepted September 22, 2016

DOI: $10.3892 /$ etm.2017.4233

\begin{abstract}
Osteoporosis is frequently asymptomatic, presenting a significant clinical and economic burden, particularly following an osteoporosis-associated fracture. Icariin has been reported to inhibit osteoporosis in vitro, and the present study investigated whether icariin also promoted bone fracture healing in ovariectomized osteoporotic (OVX) rats in vivo. A total of 30 female rats were randomly divided into three groups ( $n=10$ per group): i) Sham surgery; ii) OVX; and iii) OVX with icariin (OVX + ICA) groups. At 3 months after the ovariectomy, a unilateral cross-tibia fracture was made at the proximal right tibia. Animals were then sacrificed after 5 weeks of oral treatment. X-rays were taken at 1 week, 3 weeks and 5 weeks of treatment, and dual energy X-ray absorptiometry was used to measure the bone mineral density (BMD). Changes to the osteocalcin (BGLAP), alkaline phosphatase (ALP), tartrate-resistant acid phosphatase (TRAP) and estradiol levels in blood were measured. Callus formation and bone union were observed, the BMD was significantly higher and the BGLAP, ALP and TRAP levels were reduced, but no significant increase was observed in the blood estradiol level in the OVX + ICA group compared with the OVX group. The present findings indicate that icariin has potential as a novel alternative therapeutic agent for fracture healing in postmenopausal osteoporosis.
\end{abstract}

Correspondence to: Dr Xin-Hua Cheng, Department of Orthopedic Surgery, Renmin Hospital, Hubei University of Medicine, 39 Chaoyang Middle Road, Shiyan, Hubei 442000, P.R. China

E-mail: 33556511@qq.com

${ }^{*}$ Contributed equally

Key words: icariin, osteoporosis, fracture healing, ovariectomized osteoporotic rats

\section{Introduction}

Osteoporosis is a disease that frequently presents asymptomatically, and is typically characterized by low bone mass, microarchitectural deterioration of bone tissue and decreased bone strength $(1,2)$. Osteoporosis presents with symptoms such as low bone density and microarchitectural deterioration of bone tissue with a consequent increase in bone fragility and susceptibility to fracture (3), and with a T-score of -2.5 standard deviations (SD) below the bone mineral density (BMD) of a healthy person of the same gender (4), which is most widely measured using dual energy X-ray absorptiometry (DXA) (5). In the United States, osteoporosis affects $2 \%$ of men and $10 \%$ of women aged $\geq 50$ years old (6). Furthermore, $49 \%$ of older women and $30 \%$ of older men have osteopenia (7). Estimates indicate that $50 \%$ of women and $20 \%$ of men aged $>50$ years old will experience an osteoporosis-associated fracture. Among these osteoporosis-associated fractures, hip fracture is the most devastating of these, due to the consequent disability, mortality and costs (8). Owing to the aging of the global population, estimates suggest that the incidence of osteoporosis will double in the next 20 years (2). Consequently, an exponential increase in the numbers of fractures is anticipated, with an inevitable increased clinical and economic burden for healthcare systems.

Most strategies for treating bone loss have focused on pharmacological interventions (9); however, drug treatments may have adverse effects and poor long-term adherence, despite their effectiveness (10). Chinese herbal medicine has been used for thousands of years for the treatment of bone diseases (11). For postmenopausal women, Epimedium pubescens flavonoids are one of the most frequently used herbal compounds that is prescribed for the treatment of osteoporosis (12). Epimedium-derived phytoestrogenic flavonoids inhibit bone resorption, stimulate bone formation and prevent ovariectomy-induced osteoporosis, without resulting in uterine hyperplasia. It is suggested that these compounds have an anabolic effect on osteoporotic bone by concomitantly promoting the osteogenic differentiation of bone marrow stromal cells while suppressing adipogenic differentiation (13). Icariin $\left(\mathrm{C}_{33} \mathrm{H}_{40} \mathrm{O}_{15}\right.$; molecular weight: 676.65; Fig. 1), one of the primary active compounds within Epimedium, reportedly has an anabolic effect on the bone; it stimulates the 
proliferation of rat bone marrow stromal cells, increases the number that stain positive for osteocalcin (BGLAP) secretion, alkaline phosphatase (ALP) and enhances ALP activity, and calcium deposition levels in a dose-dependent manner $(14,15)$. In previous work, we reported that icariin inhibits osteoporosis in vitro, potentially owing to its role in increasing bone morphogenetic protein-2 (BMP-2) protein expression (16), and that icariin promotes bone formation via the BMP-2/Smad4 signal transduction pathway in the hFOB 1.19 human osteoblastic cell line (17).

The present study investigated whether icariin promotes bone fracture healing in ovariectomized osteoporotic (OVX) rats in vivo, with the intention of determining a novel method to treat osteoporosis-associated fracture.

\section{Materials and methods}

Animals and modeling method. For the present study, 30 6-month-old Sprague-Dawley (SD) female rats were obtained from Hubei University of Medicine (Shiyan, China). The rats were housed in a temperature-controlled room $\left(25^{\circ} \mathrm{C}\right)$ with constant humidity (40-50\%) and received food and water ad libitum. Rats were left for 1 week to acclimatise to their environment, which was subject to a $12 / 12 \mathrm{~h}$ light/dark cycle. These rats were randomly divided into three groups consisting of 10 rats per group, as follows: i) Sham surgery (SS); ii) OVX; and iii) OVX and icariin (OVX + ICA) groups.

Bilateral ovariectomy was performed in 20 female rats through an incision in the back, under general anesthesia with an intraperitoneal injection of $10 \%$ chloral hydrate at a dose of $3 \mathrm{ml} / \mathrm{kg}$ (Chemical Reagent Co., Shanghai, China). Approximately $1.5 \mathrm{~cm}$ of the skin, the abdominal cavity and the muscles were incised, and the ovaries were exposed (Fig. 2A). The oviduct was ligated with a silk thread and the ovariectomy was performed bilaterally (Fig. 2B), while the remaining 10 animals underwent a sham surgery in which the bilateral ovaries were examined and returned to the original position under the same protocol.

Three months after the ovariectomy, a unilateral cross-tibial fracture was made at the proximal right tibia and fixed with intramedullary nailing (diameter, $1 \mathrm{~mm}$, length, $50 \mathrm{~mm}$; Wego Medical Systems Co., Ltd, Weihai, China; Fig. 3), performed under anesthesia. All procedures were approved by the Animal Research Ethics Board at Hubei University of Medicine (Shiyan, China).

Treatment method. Icariin was obtained from the Institute of Pharmaceutical Research (Beijing, China) with a purity of $99 \%$, dissolved with $0.9 \%$ sodium chloride at a concentration of $100 \mathrm{mg} / \mathrm{ml}$. The OVX + ICA group was treated with a daily $150 \mathrm{mg} / \mathrm{kg}$ icariin, administered orally following the intramedullary fixation and right tibial fracture procedure. The SS group and OVX group received equal amounts of $0.9 \%$ sodium chloride orally.

Specimen collection. X-rays (800 mA, 150 kV, R-500; GE Healthcare Bio-Sciences, Pittsburgh, PA, USA) were taken at 1, 3 and 5 weeks after oral treatment, and dual energy X-ray absorptiometry (DSC-3000, Aloka, Tokyo, Japan) was used to measure the BMD $\left(\mathrm{mg} / \mathrm{cm}^{2}\right)$ prior to sacrifice, 5 weeks after

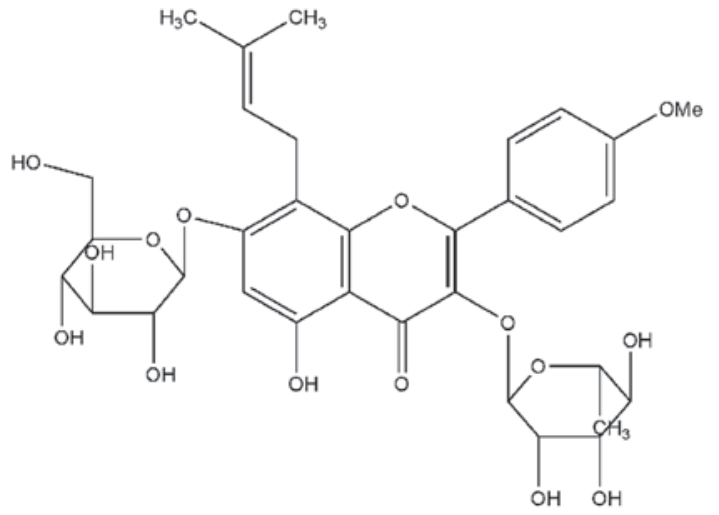

Figure 1. Chemical structure of icariin $\left(\mathrm{C}_{33} \mathrm{H}_{4} \mathrm{O}_{15}\right.$, molecular weight 676.65).
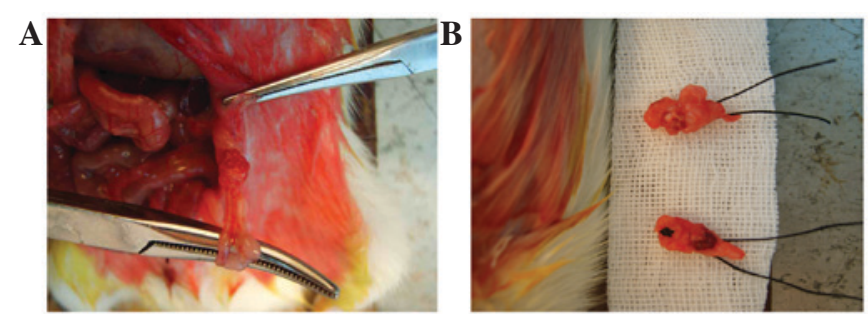

Figure 2. Following anesthetization, a bilateral ovariectomy was performed in rats. (A) Ovary was exposed and (B) each fallopian tube was completely ligated using 2-0 silk thread; bilateral ovariectomy was performed.
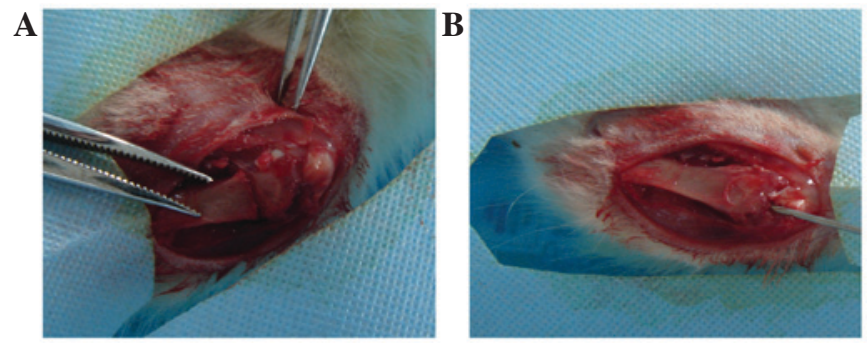

Figure 3. Three months after the ovariectomy: (A) A unilateral cross-tibial fracture was made at the proximal end of the right tibia and (B) fracture of the right tibia was fixed with an intramedullary nailing.

oral treatment. The rats were sacrificed by cervical dislocation. Blood was drawn from the inferior vena cava following sacrifice, added to anticoagulant, and was stirred at a rate of 3,000 rpm for $20 \mathrm{~min}$ to extract the blood plasma. The extracted blood plasma was stored at $-70^{\circ} \mathrm{C}$ until analysis.

Blood variable analysis. BGLAP and ALP, as bone formation markers, were each measured with an ELISA kit (BGLAP, cat. no. DSTCN0; ALP, cat. no. DY725; R\&D Systems, Inc., Minneapolis, MN, USA). Tartrate-resistant acid phosphatase (TRAP), used as a bone resorption marker, and blood estradiol levels were also measured using ELISA kit (cat. no. KGE014; R\&D Systems, Inc.).

Statistical analysis. Data are expressed as mean \pm standard deviation, and statistical analyses were performed using SPSS software, version 12.0 (SPSS, Inc., Chicago, IL, USA). One-way analysis of variance was used to assess differences 


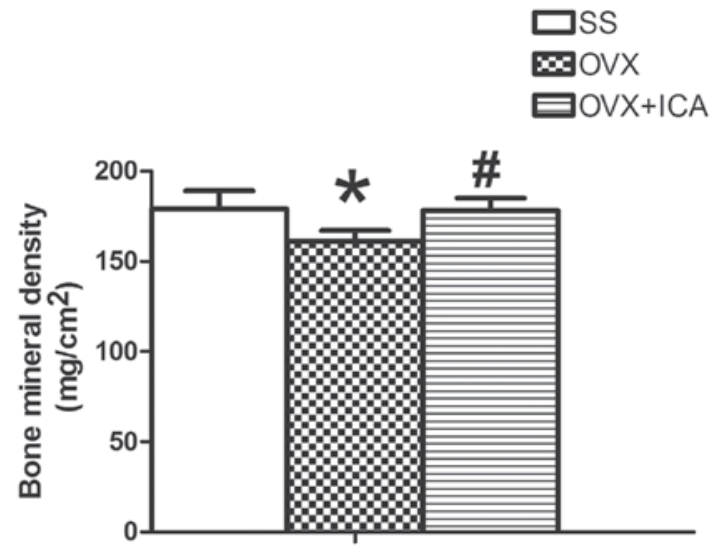

Figure 4. Bone mineral density, measured from the trabecular bone of the distal femur of the female rats. ${ }^{\mathrm{P}}<0.05$ vs. SS group; ${ }^{~} \mathrm{P}<0.05$ vs. OVX group. SS, sham surgery; OVX, ovariectomized osteoporotic; ICA, icariin.

A

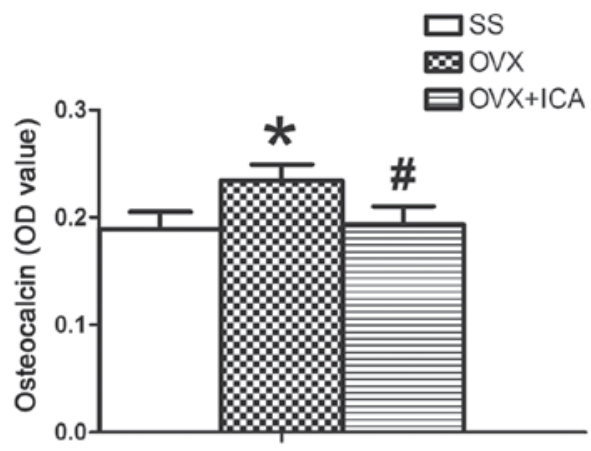

B

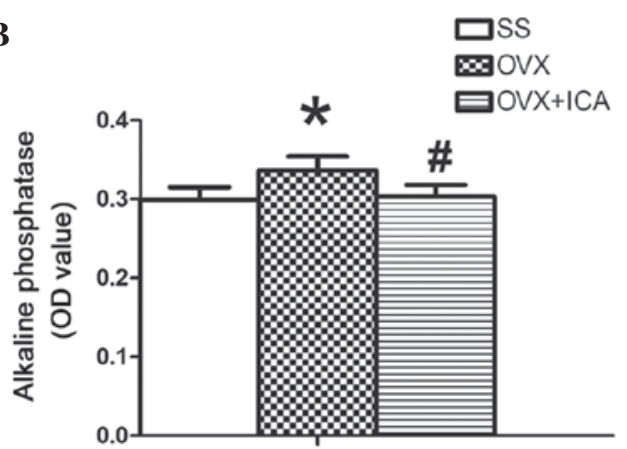

Figure 5. Effects after 5 weeks of icariin treatment on the bone formation markers (A) blood osteocalcin and (B) alkaline phosphatase. "P<0.05 vs. SS group; " $\mathrm{P}<0.05$ vs. OVX group. SS, sham surgery; OVX, ovariectomized osteoporotic; ICA, icariin; OD, optical density.

between the groups. $\mathrm{P}<0.05$ was considered to indicate a statistically significant difference.

\section{Results}

Bone mineral density is altered following icariin treatment of OVX rats. The BMD of the SS group, measured from the trabecular bone of the distal femur of the rats, was significantly higher than that of the OVX group $(\mathrm{P}<0.05)$. The BMD of OVX + ICA group was significantly higher than that of the OVX group $(\mathrm{P}<0.05)$, but was not significantly different from the BMD of the control group ( $\mathrm{P}>0.05$; Fig. 4$)$.

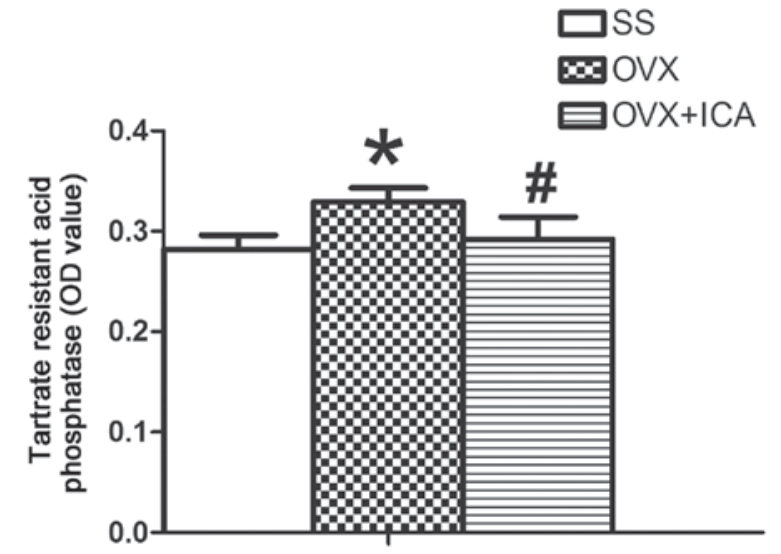

Figure 6. Effects after 5 weeks of icariin treatment on the bone resorption marker tartrate resistant acid phosphate. ${ }^{*} \mathrm{P}<0.05$ vs. SS group; ${ }^{\text {"}} \mathrm{P}<0.05$ vs. OVX group. SS, sham surgery; OVX, ovariectomized osteoporotic; ICA, icariin; OD, optical density.

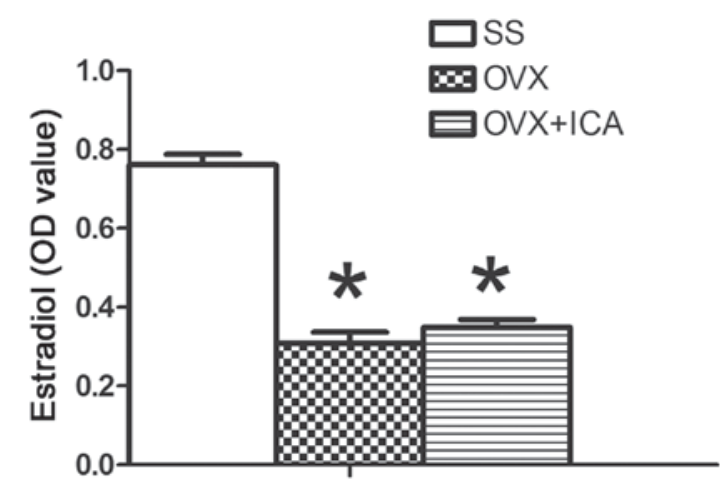

Figure 7. Blood estradiol levels after 5 weeks of icariin treatment. "P $<0.05$ vs. SS group. SS, sham surgery; OVX, ovariectomized osteoporotic; ICA, icariin; OD, optical density.

BGLAP, ALP and TRAP levels increase in OVX rats, and are rescued by icariin treatment. After 5 weeks of oral treatment, the OVX group demonstrated significantly increased BGLAP, ALP and TRAP levels compared with the SS group $(\mathrm{P}<0.05)$. The OVX + ICA group demonstrated a reduction of BGLAP, ALP and TRAP levels compared with the OVX group $(\mathrm{P}<0.05$; Figs. 5 and 6).

Changes are observed in blood estradiol levels in OVX rats, which are not rescued following icariin treatment. OVX rats exhibited a significant reduction in their blood estradiol level compared with that in the SS group $(\mathrm{P}<0.05)$. However, the OVX + ICA group exhibited no significant increase in the blood estradiol levels compared with those in the OVX group (P>0.05; Fig. 7).

$X$-rays of rat tibias reveal incomplete remodeling in OVX rats, which is improved by icariin treatment. The effects of icariin on callus formation, remodeling and bone union were observed at 1, 3 and 5 weeks after treatment. Callus formation and bone union were observed in the OVX + ICA group at 5 weeks but, in the OVX group, a small callus was formed and remodeling remained incomplete, with no union of bones (Fig. 8). 

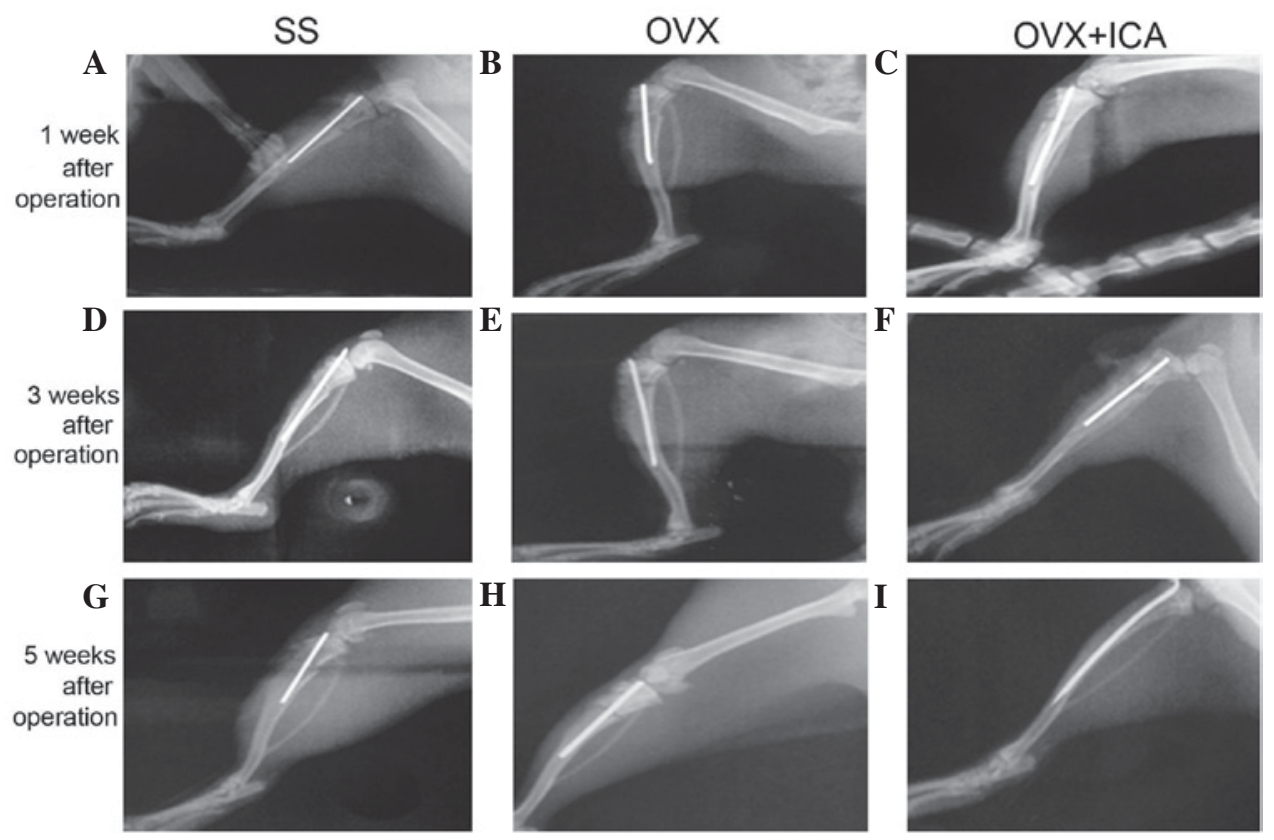

Figure 8. X-rays at (A-C) 1 week, (D-F) 3 weeks and (G-I) 5 weeks after ovariectomy in the (A, D and G) SS; (B, E and H) OVX; and (C, F and I) OVX + ICA groups, demonstrating the degree of callus formation and bone union following fracture. SS, sham surgery; OVX, ovariectomized osteoporotic; ICA, icariin

\section{Discussion}

Osteoporosis, which results from a disturbance in normal bone remodeling by increasing bone resorption relative to bone formation, is identified by a low bone mass and leads to a high risk of fractures (18). Osteoporosis is often an undiagnosed disease prior to the occurrence of fracture. Bone fragility occurs due to an excess of resorption and reduced bone formation, resulting in an increased risk of hip and vertebral fractures (19). It is reported that osteoporosis affects about 25 million individuals in the United States alone, and it is estimated that women $>50$ years old possess an $11-18 \%$ risk of suffering a hip fracture (20). Therefore, to prevent and treat the osteoporosis, medication, exercise and additional treatments such as hormone replacement therapy are commonly used $(21,22)$. Amongst these, hormone replacement therapy is the most widely used; however, previous evidence indicates that long-term treatments with these drugs may cause adverse reactions, such as an increased risk of ovarian and endometrial cancer $(23,24)$. Thus, an alternative therapeutic strategy with a proven efficacy and safety is required to prevent and treat osteoporosis. Icariin, one of the primary active ingredients of Epimedium, reportedly has an anabolic effect on bones; this may contribute to its role in the induction of osteoblast proliferation and differentiation, which results in bone formation $(25,26)$.

The ovariectomy model is a well-established animal model in osteoporosis studies (27). The OVX rat model was selected for the present study as it shares numerous similarities with postmenopausal bone loss and is recommended by the US Food and Drug Administration as a test species for evaluating the long-term skeletal safety and efficacy of osteoporosis therapies (20). Previous laboratory studies have reported that osteoporosis impairs fracture healing in early and late stages $(28,29)$. In the present study, it was investigated whether icariin promotes bone fracture healing in OVX rats in vivo; this was determined 3 months after the ovariectomy, the unilateral cross-tibia fracture was made and the fracture had been fixed with an intramedullary nailing.

Decreased BMD is one of the major factors jeopardizing bone strength, resulting in increased susceptibility to fractures (30). The present study revealed that OVX reduced BMD in the distal femurs of female SD rats, which are rich in trabecular bone, whilst treatment with icariin prevented these decreases in BMD.

BGLAP is closely bonded with hydroxyapatite and calcium in the bone, and is an established bone formation marker; this was used to predict the bone loss rate as it is indirectly involved in the activation of osteoblasts during bone generation (31). ALP is a hydrolase enzyme responsible for removing phosphate groups from many types of molecules, including nucleotides, proteins, and alkaloids (32). ALP increases during active bone formation, as ALP is a byproduct of osteoblast activity (heightened levels of which appear in Paget's disease) (33). TRAP is a glycosylated monomeric metalloprotein enzyme expressed in mammals, which may be used as a bone resorption marker. Osteopontin and bone sialoprotein, which are bone matrix phosphoproteins, are highly efficient in vitro TRAP substrates that bind to osteoclasts when phosphorylated. Upon partial dephosphorylation, osteopontin and bone sialoprotein are incapable of binding to osteoclasts (34). From this effect, it has been hypothesized that TRAP is secreted from the ruffled membrane of osteoclasts, where it dephosphorylates osteopontin and allows osteoclast migration and additional resorption to occur (35). The OVX group significantly increased the blood BGLAP, ALP and TRAP levels, while the OVX + ICA group rescued these. These results may be due to decreased estrogen increasing the number of osteoblasts and osteoclasts, or altered activity of these cells. 
The most common type of osteoporosis is the post-menopausal bone loss associated with ovarian hormone deficiency (36). Several previous studies report that estrogen is the most important hormone in maintaining bone mass and that a deficiency of this hormone is a major cause of bone loss associated with age in both genders (37-39). Notably, when the circulating estrogen level decreases, calcium in the bones rapidly decreases, and calcium loss occurs via an increase in its urinary excretion (40). In the current study, rat blood estradiol level was gauged using an ELISA kit. Those rats which experienced the menopause induced by ovariectomy demonstrated a significant decrease in blood estradiol compared with the SS group. Nian et al (41) suggested that icariin has an antiosteoporotic effect, similar to estrogen, and that it may be effective for prevention of bone fractures induced by estrogen deficiency. In the present study, the OVX + ICA group that was treated with icariin intragastrically for 5 weeks demonstrated a non-significant increase in blood estradiol compared with the OVX group. Ye et al (42) previously reported that nonconjugated forms of icaritin and desmethylicaritin, two derivatives of icariin, possess estrogen-like activity; however, icariin appeared to have no estrogenicity in the MCF-7 cell line model in vitro. Mok et al (43) revealed that icariin exerts anabolic effects in bone, possibly by activating the endoplasmic reticulum in a ligand-independent manner. In previous work, we reported that icariin inhibits osteoporosis in vitro, potentially owing to its role in increasing BMP-2 protein expression (16), and that icariin promotes bone formation via the BMP-2/Smad4 signal transduction pathway in the hFOB 1.19 human osteoblastic cell line (17).

The present study investigated the effects of icariin on callus formation, remodeling and bone union at 1, 3 and 5 weeks after treatment by examining X-rays. Callus formation and bone union increased every 2 weeks in the SS group and the fracture line was fuzzy at 5 weeks after sham surgery. In the OVX group, small calluses were formed and the fracture line was evident, revealing an absence of union. Callus formation and bone union increased temporally following icariin treatment, the fracture line was almost absent, and bone union and remodeling were observed 5 weeks after intragastric administration.

In conclusion, the present in vivo study reported that icariin attenuates the decrease in BMD in rats with osteopenia and that postfracture administration of icariin accelerates mineralization and osteogenesis and is associated with improved fracture healing. The current findings indicate that icariin has the potential to be developed as an alternative for fracture healing in postmenopausal osteoporosis. However, it should be noted that the mechanism by which icariin performs these roles remains to be examined.

\section{Acknowledgements}

The present study was supported by the National Natural Science Foundation of China (grant no. 81602867), the Hubei Provincial Department of Education (grant no. Q20132108), a Hubei Province Health and Family Planning Scientific Research Project grant (grant no. WJ2015Q042), Hubei Provincial Science and Technology Department funding (grant no. 2013CFC031).

\section{References}

1. Gallagher JC and Tella SH: Controversies in osteoporosis management: Antiresorptive therapy for preventing bone loss: When to use one or two antiresorptive agents? Clin Obstet Gynecol 56: 749-756, 2013.

2. Coughlan T and Dockery F: Osteoporosis and fracture risk in older people. Clin Med (Lond) 14: 187-191, 2014.

3. Totosy de Zepetnek JO, Giangregorio LM and Craven BC: Whole-body vibration as potential intervention for people with low bone mineral density and osteoporosis: A review. J Rehabil Res Dev 46: 529-542, 2009.

4. Pritchard JM, Giangregorio LM, Atkinson SA, Beattie KA Inglis D, Ioannidis G, Gerstein H, Punthakee Z, Adachi JD and Papaioannou A: Changes in trabecular bone microarchitecture in postmenopausal women with and without type 2 diabetes: A two year longitudinal study. BMC Musculoskelet Disord 14: 114, 2013.

5. MacIntyre NJ, Adachi JD and Webber CE: In vivo measurement of apparent trabecular bone structure of the radius in women with low bone density discriminates patients with recent wrist fracture from those without fracture. J Clin Densitom 6: 35-43, 2003.

6. Jiang X, Good LE, Spinka R and Schnatz PF: Osteoporosis screening in postmenopausal women aged 50-64 years: BMI alone compared with current screening tools. Maturitas 83: 59-64, 2016.

7. Looker AC, Melton LJ III, Harris TB, Borrud LG and Shepherd JA: Prevalence and trends in low femur bone density among older US adults: NHANES 2005-2006 compared with NHANES III. J Bone Miner Res 25: 64-71, 2010.

8. Holvik K, Ahmed LA, Forsmo S, Gjesdal CG, Grimnes G, Samuelsen SO, Schei B, Blomhoff R, Tell GS and Meyer HE: No increase in risk of hip fracture at high serum retinol concentrations in community-dwelling older Norwegians: The Norwegian Epidemiologic Osteoporosis Studies. Am J Clin Nutr 102: 1289-1296. 2015

9. Hamdy RC, Baim S, Broy SB, Lewiecki EM, Morgan SL, Tanner SB and Williamson HF: Algorithm for the management of osteoporosis. South Med J 103: 1009-1015, 2010.

10. Green J, Czanner G, Reeves G, Watson J, Wise L and Beral V: Oral bisphosphonates and risk of cancer of oesophagus, stomach and colorectum: Case-control analysis within a UK primary care cohort. BMJ 341: c4444, 2010.

11. Wang WL, Sheu SY, Chen YS, Kao ST, Fu YT, Kuo TF, Chen KY and Yao $\mathrm{CH}$ : Enhanced bone tissue regeneration by porous gelatin composites loaded with the Chinese herbal decoction Danggui Buxue Tang. PLoS One 10: e0131999, 2015.

12. Zhang G, Qin L and Shi Y: Epimedium-derived phytoestrogen flavonoids exert beneficial effect on preventing bone loss in late postmenopausal women: A 24-month randomized, double-blind and placebo-controlled trial. J Bone Miner Res 22: 1072-1079, 2007.

13. Peng S, Zhang G, He Y, Wang X, Leung P, Leung K and Qin L: Epimedium-derived flavonoids promote osteoblastogenesis and suppress adipogenesis in bone marrow stromal cells while exerting an anabolic effect on osteoporotic bone. Bone 45: 534-544, 2009.

14. Xu JH, Yao M, Ye J, Wang GD, Wang J, Cui XJ and Mo W: Bone mass improved effect of icariin for postmenopausal osteoporosis in ovariectomy-induced rats: A meta-analysis and systematic review. Menopause 23: 1152-1157, 2016.

15. Chen KM, Ge BF, Ma HP, Liu XY, Bai MH and Wang Y: Icariin, a flavonoid from the herb epimedium enhances the osteogenic differentiation of rat primary bone marrow stromal cells Pharmazie 60: 939-942, 2005.

16. Cao H, Ke Y, Zhang Y, Zhang CJ, Qian W and Zhang GL: Icariin stimulates MC3T3-E1 cell proliferation and differentiation through up-regulation of bone morphogenetic protein- 2 . Int $\mathrm{J}$ Mol Med 29: 435-439, 2012.

17. Liang W, Lin M, Li X, Li C, Gao B, Gan H, Yang Z, Lin X, Liao $L$ and Yang M: Icariin promotes bone formation via the BMP-2/Smad4 signal transduction pathway in the hFOB 1.19 human osteoblastic cell line. Int J Mol Med 30: 889-895, 2012.

18. Zhang Z, Xiang L, Bai D, Fu X, Wang W, Li Y, Liu H, Pan J, Li Y, Xiao GG and Ju D: Treatment with rhizoma dioscoreae extract has protective effect on osteopenia in ovariectomized rats. ScientificWorldJournal 2014: 645975, 2014.

19. de Laet CE, van der Klift M, Hofman A and Pols HA: Osteoporosis in men and women: A story about bone mineral density thresholds and hip fracture risk. J Bone Miner Res 17: 2231-2236, 2002. 
20. Choi JS, Kim JW, Kim KY, Cho HR, Choi IS and Ku SK: Antiosteoporotic effects of polycan in combination with calcium lactate-gluconate in ovariectomized rats. Exp Ther Med 8: 957-967, 2014

21. Milat F and Ebeling PR: Osteoporosis treatment: A missed opportunity. Med J Aust 205: 185-190, 2016.

22. Henriksen K, Byrjalsen I, Andersen JR, Bihlet AR, Russo LA, Alexandersen P, Valter I, Qvist P, Lau E, Riis BJ, et al; SMC021 Investigators: A randomized, double-blind, multicenter, placebo-controlled study to evaluate the efficacy and safety of oral salmon calcitonin in the treatment of osteoporosis in postmenopausal women taking calcium and vitamin D. Bone 91: 122-129, 2016.

23. Strom BL, Schinnar R, Weber AL, Bunin G, Berlin JA, Baumgarten M, DeMichele A, Rubin SC, Berlin M, Troxel AB and Rebbeck TR: Case-control study of postmenopausal hormone replacement therapy and endometrial cancer. Am J Epidemiol 164: 775-786, 2006.

24. Rossing MA, Cushing-Haugen KL, Wicklund KG, Doherty JA and Weiss NS: Menopausal hormone therapy and risk of epithelial ovarian cancer. Cancer Epidemiol Biomarkers Prev 16: 2548-2556, 2007.

25. Kapoor S: Icariin and its emerging role in the treatment of osteoporosis. Chin Med J (Engl) 126: 400, 2013.

26. Luo Z, Liu M, Sun L and Rui F: Icariin recovers the osteogenic differentiation and bone formation of bone marrow stromal cells from a rat model of estrogen deficiency-induced osteoporosis. Mol Med Rep 12: 382-388, 2015.

27. Li CL, Liu XL, Cai WX, Lu WW, Zwahlen RA and Zheng LW: Effect of ovariectomy on stimulating intracortical remodeling in rats. Biomed Res Int 2014: 421431, 2014.

28. Namkung-Matthai H, Appleyard R, Jansen J, Hao Lin J, Maastricht S, Swain M, Mason RS, Murrell GA, Diwan AD and Diamond T: Osteoporosis influences the early period of fracture healing in a rat osteoporotic model. Bone 28: 80-86, 2001.

29. Kubo T, Shiga T, Hashimoto J, Yoshioka M, Honjo H, Urabe M, Kitajima I, Semba I and Hirasawa Y: Osteoporosis influences the late period of fracture healing in a rat model prepared by ovariectomy and low calcium diet. J Steroid Biochem Mol Biol 68 : 197-202, 1999.

30. Park JA, Ha SK, Kang TH, Oh MS, Cho MH, Lee SY, Park JH and Kim SY: Protective effect of apigenin on ovariectomy-induced bone loss in rats. Life Sci 82: 1217-1223, 2008.

31. Johansen JS, Riis BJ, Delmas PD and Christiansen C: Plasma BGP: An indicator of spontaneous bone loss and of the effect of oestrogen treatment in postmenopausal women. Eur J Clin Invest 18: 191-195, 1988.
32. Sarvari BK, Sankara Mahadev D, Rupa S and Mastan SA: Detection of bone metastases in breast cancer (BC) patients by serum tartrate-resistant acid phosphatase $5 \mathrm{~b}$ (TRACP 5b), a bone resorption marker and serum alkaline phosphatase (ALP), a bone formation marker, in lieu of whole body skeletal scintigraphy with Technetium99m MDP. Indian J Clin Biochem 30: 66-71, 2015.

33. Pruessner HT: Detecting celiac disease in your patients. Am Fam Physician 57: 1023-1034, 1998.

34. Wu G, Guo JJ, Ma ZY, Wang J, Zhou ZW and Wang Y: Correlation between calcification and bone sialoprotein and osteopontin in papillary thyroid carcinoma. Int J Clin Exp Pathol 8: 2010-2017, 2015.

35. Ek-Rylander B,Flores M,Wendel M,Heinegard D and Andersson G: Dephosphorylation of osteopontin and bone sialoprotein by osteoclastic tartrate-resistant acid phosphatase. Modulation of osteoclast adhesion in vitro. J Biol Chem 269: 14853-14856, 1994.

36. Rugpolmuang L and Waikakul S: Effect of a short-term treatment with once-a-week medication of alendronate $70 \mathrm{mg}$ on bone turnover markers in postmenopausal women with osteoporosis. J Med Assoc Thai 98 (Suppl 8): S70-S75, 2015.

37. Xu F, Ding Y, Guo Y, Liu B, Kou Z, Xiao W and Zhu J: Anti-osteoporosis effect of Epimedium via an estrogen-like mechanism based on a system-level approach. J Ethnopharmacol 177: 148-160, 2016.

38. Bordinhon M, Müller SS and Silva MD: Clinical, biomechanical and histological study on oophorectomy induced menopause. Acta Ortop Bras 22: 260-263, 2014.

39. Du Z, Steck R, Doan N, Woodruff MA, Ivanovski S and Xiao Y: Estrogen deficiency-associated bone loss in the maxilla: A methodology to quantify the changes in the maxillary intra-radicular alveolar bone in an ovariectomized rat osteoporosis model. Tissue Eng Part C Methods 21: 458-466, 2015.

40. Hattori S, Agata U, Park JH, Iimura Y, Tokuda S, Ezawa I and Omi N: The relationship between salivary calcium concentration and differences in bone mineral density level in female rats. J Nutr Sci Vitaminol (Tokyo) 60: 152-158, 2014.

41. Nian H, Ma MH, Nian SS and Xu LL: Antiosteoporotic activity of icariin in ovariectomized rats. Phytomedicine 16: 320-326, 2009.

42. Ye HY and Lou YJ: Estrogenic effects of two derivatives of icariin on human breast cancer MCF-7 cells. Phytomedicine 12: 735-741, 2005.

43. Mok SK, Chen WF, Lai WP, Leung PC, Wang XL, Yao XS and Wong MS: Icariin protects against bone loss induced by oestrogen deficiency and activates oestrogen receptor-dependent osteoblastic functions in UMR 106 cells. Br J Pharmacol 159: 939-949, 2010. 